\title{
Numerical Simulation of Molten Metal Flow Produced by Induction MHD Pump Using Rotating Twisted Magnetic Field*
}

\author{
Tsutomu ANDO**, Kazuyuki UENO*** and Keisuke SAWADA***
}

\begin{abstract}
Numerical simulation at the same condition as an experiment is carried out under the magnetic Stokes approximation for small shielding parameter. Results of the simulation compensate for the information of molten metal flow that we could not directly obtain in the experiment. In this paper, we study the molten metal flow at a starting condition and quasi-steady state. Besides, the energy conversion in the MHD pump is discussed. The simulation result shows that the proposed MHD pump causes the spiral induced current in a molten gallium and produces an axial flow with swirl. At quasi-steady state, it is confirmed that the centrifugal force by the excessive swirl flow produces high pressure at a duct wall and low pressure around the central axis. Since the excessive swirl flow results in large viscous dissipation, the mechanical power output of the pump uses only about $1 \%$ of the mechanical energy production in the molten gallium.
\end{abstract}

Key Words: Magnetohydrodynamics, Fluid Machinery, Computational Fluid Dynamics, Finite Difference Method, Metal Flow, Electromagnetic Pumps, Induction Machine, Molten Metal, Rotating Twisted Magnetic Field, Helical Coils

\section{Introduction}

One of the major advantages of electromagnetic force is its direct action on objects without any contact. In recent years, applications of electromagnetic force are developed in the steelmaking process handling molten steel of over $1500^{\circ} \mathrm{C}^{(1),(2)}$. Until today, however, there is no electromagnetic pump applicable to high-temperature molten metals, such as molten steel. At present, one of the most familiar conventional induction pump is the annular linear induction pump (ALIP) ${ }^{(3),(4)}$. It has an iron core installed in the duct so that the magnetic field may traverse the flow. However the iron core loses the ferromagnetic property when temperature is higher than its Curie point. Therefore, the ALIP are not applicable to hightemperature molten metals.

The authors have proposed and studied a new type MHD pump using a rotating twisted magnetic field, applicable to high-temperature molten metals ${ }^{(5)-(8)}$. The stator of our proposed machine generates the rotating twisted

\footnotetext{
* Received 31st January, 2005 (No. 05-4009)

** Department of Advanced Materials Science, The University of Tokyo, Kashiwa 277-8561, Japan. E-mail: tando@k.u-tokyo.ac.jp

*** Department of Aerospace Engineering, Tohoku University, Sendai 980-8579, Japan
}

magnetic field by a helical coil. This pump does not need the iron core in the duct because the rotating twisted magnetic field can traverse the center of the duct. This field gives axial thrust to a secondary conductor as well as rotational torque. Hence, the proposed machine has both properties of the linear pump and the rotary stirrer. In the previous paper ${ }^{(8)}$, we reported the result of circulation experiment using molten gallium of about $50^{\circ} \mathrm{C}$ and evaluated the basic performance of the proposed MHD pump. However, we could not exactly grasp the flow of molten gallium in the proposed MHD pump because only flow rate and pressure were measured in the experiment.

The present paper shows the results of the MHD numerical simulation that we carried out. Here, we performed this simulation under the approximation of small magnetic Reynolds number $R_{m} \ll 1$ and small shielding parameter $R_{\omega} \ll 1$. In this paper, we call the approximation of $R_{\omega} \ll 1$ the "magnetic Stokes approximation" although this name is not well established as the academic nomenclature. Under the magnetic Stokes approximation, we can ignore the effect of secondary magnetic field generated by the induced current in molten metal. As a consequence, magnetic field can be obtained before calculating of flow field, and it has no skin effect in this condition. Meanwhile, this simulation does not exactly take account of the influence of the turbulence, though the molten gal- 
lium flow in the experiment is suspected to be a turbulent flow. Although the present simulation neglects or underestimates the above-mentioned factors, we believe that the very useful knowledge is obtained from this simulation and it compensates for the information of molten gallium flow that we could not directly obtain in the experiment.

The present paper has two main objects. The first object is to obtain the molten gallium flow at a starting condition and quasi-steady state. The second object is to obtain the energy conversion at quasi-steady state. In order to obtain them, numerical simulation of axisymmetric flow was carried out under the magnetic Stokes approximation. Three components of the velocity vector including swirl flow were obtained as functions of $r$ and $z$. The pumping condition of the simulation is given by the input current $\bar{I}=457 \mathrm{~A}$ and frequency $f=50 \mathrm{~Hz}$ which are the same condition as one case of the experiments in the previous $\operatorname{paper}^{(8)}$.

\section{Overview of the Proposed MHD Pump and Calcu- lation Domain}

Figure 1 shows the stator windings and the magnetic field generated by the stator of the proposed pump. The direction of the field vector changes with $z$. This magnetic field rotates at a constant speed keeping the spatial distribution. Detail of specification of the proposed pump is shown in Ref. (8).

Figure 2 shows duct system including the proposed pump. It is the same as the experimental apparatus of the previous paper ${ }^{(8)}$. It has two pressure transducers at the inlet and the outlet of the pump. The range of $0 \leq a_{2}$, $-z_{0} \leq z \leq z_{0}$ is a calculation domain for the flow field. It includes two measurement points of pressure.

The notation $z_{p}$ in Fig. 2 indicates the position of the perforated plate. They were mounted in the duct in order to eliminate the swirl effect on the pressure measurement ${ }^{(8)}$. In this simulation, we assume $u_{\theta}=0$ in the area of $z \geq\left|z_{p}\right|$.

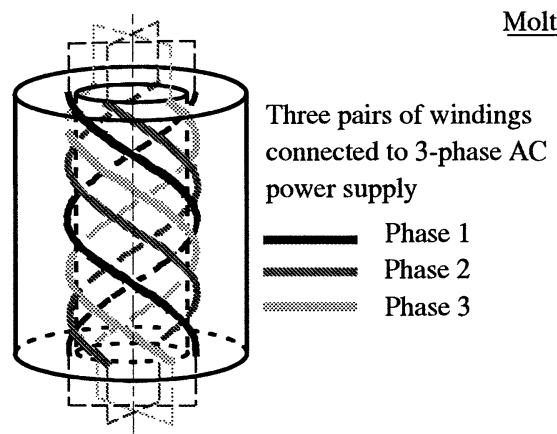

(a)

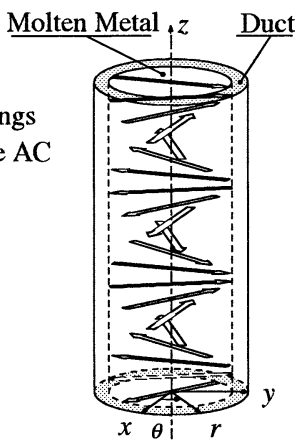

(b)
Fig. 1 Windings and magnetic field. (a) Stator of the induction pump for molten metals: three pairs of helical windings. (b) Rotating twisted magnetic field
Calculation domain for the magnetic field is $0 \leq r_{M}$, $-z_{M} \leq z \leq z_{M}$ which is shown by the broken line in Fig. 2 . It was confirmed that the extent of this domain is sufficient for calculating the magnetic flux density in the proposed pump.

\section{Equations and Calculation Method}

\subsection{Magnetic scalar potential in case of $R_{\omega} \ll 1$}

Extending the expression with complex amplitude, which is used in a usual alternating electromagnetic field, we give the cylindrical-coordinate component of the magnetic flux density as follows.

$$
\left\{\begin{array}{l}
\tilde{B}_{r}=\hat{B}_{r}(r, z) \exp [i(\omega t-\theta)], \\
\tilde{B}_{\theta}=\hat{B}_{\theta}(r, z) \exp [i(\omega t-\theta)], \\
\tilde{B}_{z}=\hat{B}_{z}(r, z) \exp [i(\omega t-\theta)],
\end{array}\right.
$$

where we put " " onto the complex functions and " " onto the complex amplitudes which are introduced by the separation of variables. Here, $\omega(=2 \pi f)$ denotes angular frequency and $i$ indicates the imaginary unit, that is $\sqrt{-1}$.

Since $|\boldsymbol{j}| /\left(|\boldsymbol{H}| / a_{2}\right)$ is estimated to be $R_{\omega}=\sigma \mu \omega a_{2}^{2}$ in the secondary conductor, Ampére's law is approximated to $\nabla \times \boldsymbol{H}=\mathbf{0}$ under the assumption of $R_{\omega} \ll 1$. Here, $\sigma$ and $\mu$ denote the electric conductivity and the permeability. Using the relation of $\boldsymbol{B}=\mu \boldsymbol{H}$, we obtain

$$
\nabla \times \boldsymbol{B}=\mathbf{0} .
$$

This is often called magnetic Stokes approximation owing to the analogy between the Navier-Stokes equation and the induction equation.

Taking Eq. (2) into account, we introduce a complex scalar potential for magnetic field $\tilde{\psi}$ and define the magnetic flux density as follows:

$$
\tilde{\boldsymbol{B}}=-\nabla \tilde{\psi} .
$$

Substituting Eq. (3) for Gauss's law, $\nabla \cdot \boldsymbol{B}=0$, we obtain the Laplace's equation for $\tilde{\psi}$ :

$$
\frac{1}{r} \frac{\partial}{\partial r}\left(r \frac{\partial \tilde{\psi}}{\partial r}\right)+\frac{1}{r^{2}} \frac{\partial^{2} \tilde{\psi}}{\partial \theta^{2}}+\frac{\partial^{2} \tilde{\psi}}{\partial z^{2}}=0
$$

We assume $\tilde{\psi}$ in the following form:

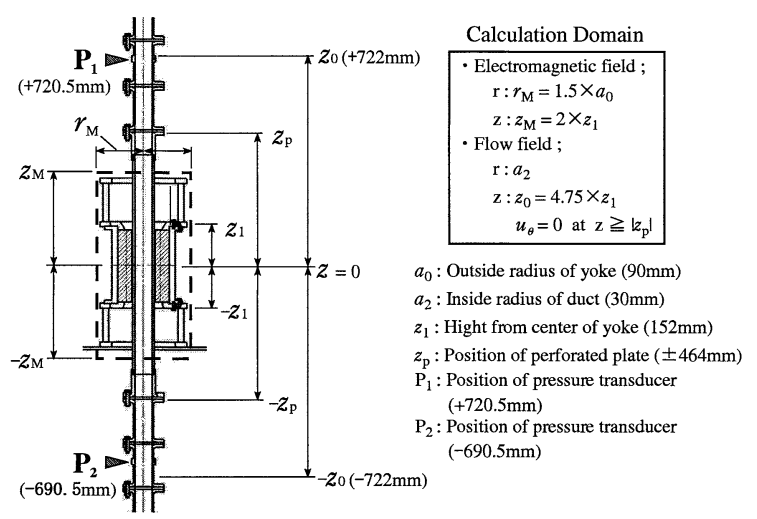

Fig. 2 Calculation domain 


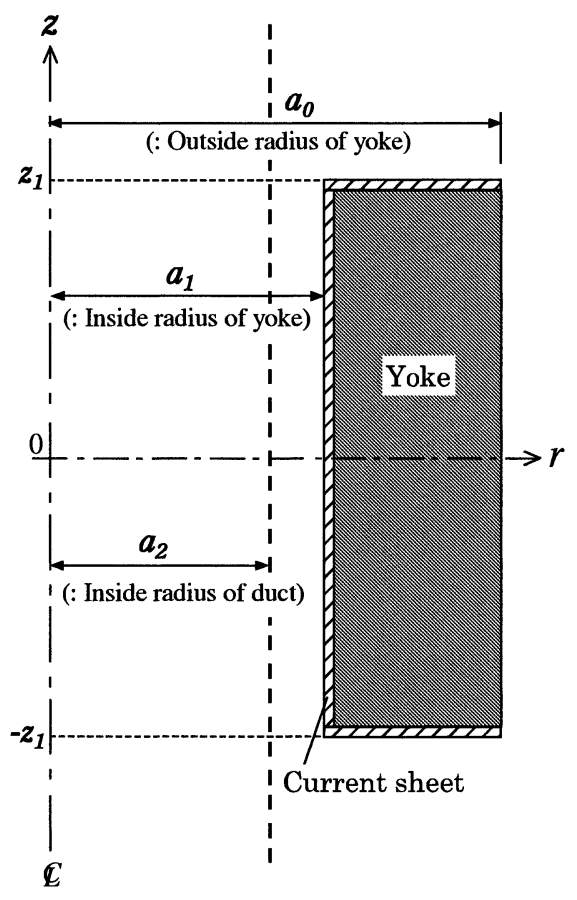

Fig. 3 Diagram of current sheet of the proposed MHD pump

$$
\tilde{\psi}(r, \theta, z ; t)=\hat{\psi}_{r z}(r, z) \cdot \exp [i(\omega t-\theta)] .
$$

Substituting Eq. (5) for Eq. (4), we obtain the partial differential equation of the $r-z$ two-dimension:

$$
r \frac{\partial}{\partial r}\left(r \frac{\partial \hat{\psi}_{r z}}{\partial r}\right)+r^{2} \frac{\partial^{2} \hat{\psi}_{r z}}{\partial z^{2}}=\hat{\psi}_{r z}
$$

We obtain $\hat{\psi}_{r z}$ at the grid points on $r-z$ plane by finite difference method.

The boundary conditions of the magnetic scalar potential $\hat{\psi}_{r z}$ are obtained from a current sheet analysis. The current sheet model is shown in Fig. 3. These boundary conditions of $\hat{\psi}_{r z}$ are shown in the following equations.

- Inner surface of the yoke: $-z_{1} \leq z \leq z_{1}, r=a_{1}$,

$$
\hat{\psi}_{r z}=-i \sqrt{2} \mu_{0} \bar{I} N \exp [i(-k z)] \text {. }
$$

- Upper surface of the yoke: $z=z_{1}, a_{1} \leq r \leq a_{0}$,

$$
\hat{\psi}_{r z}=-i \frac{\sqrt{2} \mu_{0} \bar{I} N}{a_{1}} \frac{r\left(\sqrt{a_{0}}-\sqrt{r}\right)}{\sqrt{a_{0}}-\sqrt{a_{1}}} \exp \left[i\left(-k z_{1}\right)\right] \text {. }
$$

- Lower surface of the yoke: $z=-z_{1}, a_{1} \leq r \leq a_{0}$,

$$
\hat{\psi}_{r z}=-i \frac{\sqrt{2} \mu_{0} \bar{I} N}{a_{1}} \frac{r\left(\sqrt{a_{0}}-\sqrt{r}\right)}{\sqrt{a_{0}}-\sqrt{a_{1}}} \exp \left[i\left(k z_{1}\right)\right] \text {. }
$$

- Outer surface of the yoke: $-z_{1} \leq z \leq z_{1}, r=a_{0}$,

$$
\hat{\psi}_{r z}=0 \text {. }
$$

- Central axis: $-z_{M} \leq z \leq z_{M}, r=0$,

$$
\hat{\psi}_{r z}=0 \text {. }
$$

- Outside boundary: $z= \pm z_{M}, 0 \leq r \leq r_{M}$ and $-z_{M} \leq$ $z \leq z_{M}, r=r_{M}$

$$
\hat{\psi}_{r z}=0 \text {. }
$$

Here, $k$ denotes the wave number of $z$-direction of magnetic field. $r_{M}$ and $z_{M}$ denote the maximum points of $r$ and $z$-direction in which the magnetic field is calculated. The derivation of the boundary condition of the magnetic field is shown in Appendix.

\subsection{Electric potential}

Now we assume the electric field $\boldsymbol{E}$ in the following form:

$$
\left\{\begin{array}{l}
\tilde{E}_{r}=-\frac{\partial \tilde{\phi}}{\partial r}-r \omega \tilde{B}_{z}, \\
\tilde{E}_{\theta}=-\frac{1}{r} \frac{\partial \tilde{\phi}}{\partial \theta} \\
\tilde{E}_{z}=-\frac{\partial \tilde{\phi}}{\partial z}+r \omega \tilde{B}_{r} .
\end{array}\right.
$$

In a similar to the magnetic scalar potential, we assume $\tilde{\phi}$ in the following form:

$$
\tilde{\phi}(r, \theta, z ; t)=\hat{\phi}_{r z}(r, z) \cdot \exp [i(\omega t-\theta)] .
$$

Electric field given by Eqs. (13) and (14) automatically satisfy Faraday's law.

As for the induced current, we also use the expression represented by a complex amplitude. Each component of the extended Ohm's equation, $\boldsymbol{j}=\sigma(\boldsymbol{E}+\boldsymbol{u} \times \boldsymbol{B})$, is written as

$$
\left\{\begin{array}{l}
\tilde{j}_{r}=\sigma\left[-\frac{\partial \tilde{\phi}}{\partial r}-\left(r \omega-u_{\theta}\right) \tilde{B}_{z}-u_{z} \tilde{B}_{\theta}\right], \\
\tilde{j}_{\theta}=\sigma\left[-\frac{1}{r} \frac{\partial \tilde{\phi}}{\partial \theta}+u_{z} \tilde{B}_{r}-u_{r} \tilde{B}_{z}\right], \\
\tilde{j}_{z}=\sigma\left[-\frac{\partial \tilde{\phi}}{\partial z}+\left(r \omega-u_{\theta}\right) \tilde{B}_{r}+u_{r} \tilde{B}_{\theta}\right] .
\end{array}\right.
$$

Substituting Eqs. (15) for $\nabla \cdot \boldsymbol{j}=0$, we obtain the following partial differential equation of $r-z$ two-dimension:

$$
\begin{aligned}
& \frac{1}{r} \frac{\partial}{\partial r}\left(r \frac{\partial \hat{\phi}_{r z}}{\partial r}\right)+\frac{\partial^{2} \hat{\phi}_{r z}}{\partial z^{2}}-\frac{1}{r^{2}} \hat{\phi}_{r z} \\
& \quad=-\frac{1}{r} \frac{\partial}{\partial r}\left[r\left\{\left(r \omega-u_{\theta}\right) \hat{B}_{z}+u_{z} \hat{B}_{\theta}\right\}\right] \\
& \quad+\frac{\partial}{\partial r}\left[\left(r \omega-u_{\theta}\right) \hat{B}_{r}+u_{r} \hat{B}_{\theta}\right] \\
& \quad-\frac{i}{r}\left(u_{z} \hat{B}_{r}-u_{r} \hat{B}_{z}\right) .
\end{aligned}
$$

We obtain $\hat{\phi}_{r z}$ at the grid points on $r-z$ plane by finite difference method.

Because of $\tilde{j}_{r}=0$ at the inner wall of the duct, the boundary condition of the electric potential is derived from $\boldsymbol{u}=\mathbf{0}$ and the first equation of Eq. (15):

$$
\frac{\partial \hat{\phi}_{r z}}{\partial r}+r \omega \hat{B}_{z}=0 \quad \text { at } \quad r=a_{2} .
$$

\subsection{Lorentz force}

Supposing an axisymmetric flow, the circumferential average of Lorentz force

$$
\begin{aligned}
\langle\boldsymbol{F}\rangle & =\frac{1}{2 \pi} \int_{0}^{2 \pi}(\operatorname{Re}[\tilde{\boldsymbol{j}}] \times \operatorname{Re}[\tilde{\boldsymbol{B}}]) d \theta \\
& =\frac{1}{2} \operatorname{Re}\left[\hat{\boldsymbol{j}} \times \hat{\boldsymbol{B}}^{*}\right]
\end{aligned}
$$


is the same as the time average of it, where $\hat{\boldsymbol{B}}^{*}$ indicates the complex conjugate of $\hat{\boldsymbol{B}}$. The complex amplitudes $\hat{\boldsymbol{B}}$ and $\hat{j}$ are given by the following equations, respectively:

$$
\begin{aligned}
& \left\{\begin{array}{l}
\hat{B}_{r}=-\frac{\partial \hat{\psi}_{r z}}{\partial r}, \\
\hat{B}_{\theta}=i \frac{1}{r} \hat{\psi}_{r z}, \\
\hat{B}_{z}=-\frac{\partial \hat{\psi}_{r z}}{\partial z} .
\end{array}\right. \\
& \left\{\begin{array}{l}
\hat{j}_{r}=\sigma\left[-\frac{\partial \hat{\phi}_{r z}}{\partial r}-\left(r \omega-u_{\theta}\right) \hat{B}_{z}-u_{z} \hat{B}_{\theta}\right], \\
\hat{j}_{\theta}=\sigma\left[i \frac{1}{r} \hat{\phi}_{r z}+u_{z} \hat{B}_{r}-u_{r} \hat{B}_{z}\right], \\
\hat{j}_{z}=\sigma\left[-\frac{\partial \hat{\phi}_{r z}}{\partial z}+\left(r \omega-u_{\theta}\right) \hat{B}_{r}+u_{r} \hat{B}_{\theta}\right] .
\end{array}\right.
\end{aligned}
$$

\section{4 Flow field}

The Navier-Stokes equation of conducting fluid is written as

$$
\rho\left(\frac{\partial \boldsymbol{u}}{\partial t}+(\boldsymbol{u} \cdot \nabla) \boldsymbol{u}\right)=-\nabla p+\eta \nabla^{2} \boldsymbol{u}+\boldsymbol{j} \times \boldsymbol{B},
$$

where $\rho$ and $\eta$ are the density and the viscosity of conducting fluid, respectively. We assume an axisymmetric flow:

$$
\frac{\partial \boldsymbol{u}}{\partial \theta}=\mathbf{0}, \quad \frac{\partial p}{\partial \theta}=0 .
$$

The law of mass conservation is written as

$$
\frac{1}{r} \frac{\partial\left(r u_{r}\right)}{\partial r}+\frac{\partial u_{z}}{\partial z}=0
$$

We use the Marker-and-Cell (MAC) $\operatorname{method}^{(9)}$ on the present simulation. And here, an irregular rectangular staggered mesh system was employed. We used the third order upwind scheme ${ }^{(10)}$ for the nonlinear terms to obtain numerically stable solution.

The present simulation was carried out at the same condition as one case of the experiment ${ }^{(8)}$. The input current $\bar{I}$ is $457 \mathrm{~A}$ and frequency $f$ is $50 \mathrm{~Hz}$. The conducting fluid is molten gallium at $50^{\circ} \mathrm{C}$.

We assume the periodic boundary condition at the outlet and the inlet of the calculation domain, though the pressure has difference $\Delta p$ between them. This pressure difference $\Delta p$ is given by

$$
\Delta p=\beta Q^{2}, \quad Q=2 \pi \int_{0}^{a_{2}} u_{z} r d r,
$$

where the coefficient $\beta$ is given by the empirical value $89.1 \mathrm{~Pa} \cdot \mathrm{min}^{2} / \mathrm{L}^{2}$ obtained in the experiment ${ }^{(8)}$. This relation is explained as pressure loss of fluidic load at the opposite side of the molten gallium circulation system.

\section{Results of Numerical Analysis under Magnetic Stokes Approximation}

\subsection{Magnetic field}

First, we obtained numerical solution $\hat{\psi}_{r z}$ of Eqs. (6) (12) on the $r-z$ plane. Furthermore, we produced threedimensional distributions of the magnetic flux density by Eqs. (3) and (5). As a result of examination of the threedimensional field distribution, it was confirmed that the obtained magnetic field realized the rotating twisted magnetic field.

Next, we compared the value of the numerical simulation with the measurement value of the previous paper $^{(8)}$, as shown in Table 1. The magnitude of numerical solution of the magnetic flux density is different from the measurement value because the current sheet used in the calculation is an ideal model, which is the infinitesimal thin current concentrated on the inner surface of the yoke. In actual, a coil has the width of windings. In this experimental stator, the inner radius of coil is $40 \mathrm{~mm}$ and the inner radius of yoke is $62.5 \mathrm{~mm}$. In the present result, the calculated value is about $7 \%$ smaller than the measured value in the experiment. Therefore, we multiply the input current of resource parameter by the correction factor 1.08 just only first.

\section{2 Lorentz force and flow field}

Two-dimensional axisymmetric simulations were carried out to investigate the molten gallium flow in the proposed MHD pump. Before the MHD simulation, we confirmed to realize the Hagen-Poiseuille flow when $\boldsymbol{F}=\mathbf{0}$.

4.2.1 Results at a starting condition The present section discusses the result of simulation at $t=$ $0.01 \mathrm{~s}$. Figure 4 shows the streamtubes and the pressure

\begin{tabular}{|c|c|c|c|c|c|c|c|}
\hline \multirow{2}{*}{$\begin{array}{c}\text { Current } \\
\bar{I} \\
{[\mathrm{~A}]}\end{array}$} & & \multirow{2}{*}{$\begin{array}{c}r=0 \mathrm{~mm} \\
\bar{B}_{x}=\bar{B}_{y} \\
{[\mathrm{mT}]}\end{array}$} & \multicolumn{2}{|c|}{$r=20 \mathrm{~mm}$} & \multicolumn{2}{|c|}{$r=32 \mathrm{~mm}$} & \multirow{2}{*}{$\begin{array}{c}\text { Remarks } \\
\text { (Magnification) }\end{array}$} \\
\hline & & & $\begin{array}{c}\bar{B}_{r} \\
{[\mathrm{mT}]}\end{array}$ & $\begin{array}{c}\bar{B}_{\theta} \\
{[\mathrm{mT}]}\end{array}$ & $\begin{array}{c}\bar{B}_{r} \\
{[\mathrm{mT}]}\end{array}$ & $\begin{array}{c}\bar{B}_{\theta} \\
{[\mathrm{mT}]}\end{array}$ & \\
\hline \multirow{2}{*}{30} & Measurement ${ }^{\dagger}$ & 4.5 & 4.5 & 4.4 & 5.0 & 4.6 & \\
\hline & Analysis ${ }^{\S}$ & 4.33 & 4.56 & 4.44 & 4.99 & 4.59 & $(30 \mathrm{~A} \times 108 \%)$ \\
\hline \multirow{2}{*}{457} & Extrapolated value & 68.6 & 68.6 & 67.0 & 76.2 & 70.1 & Measurement $\times 457 / 30$ \\
\hline & Analysis $\S$ & 65.3 & 68.8 & 67.0 & 75.1 & 69.1 & $(457 \mathrm{~A} \times 108 \%)$ \\
\hline
\end{tabular}
distribution of molten gallium in the duct. Figure 4 (a) shows that Lorentz force practically acts on the range of the height of the stator. Figure 4 (b) shows that pressure rise is generated between the inlet and the outlet of the

Table 1 Comparison of magnetic flux density on the cross section at $z=0 \mathrm{~mm}$

$\dagger$ Measurement value in ref.(8).

$\S$ Numerical analysis using current sheet with coil-end. 


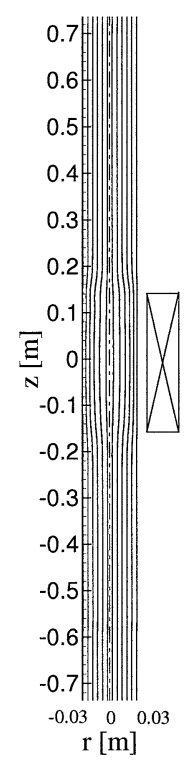

(a) Streamtubes (b) Pressure distribution

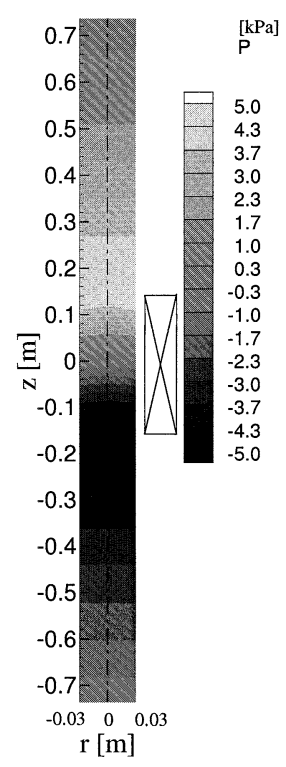

Fig. 4 Streamtubes and Pressure distribution at $t=0.01 \mathrm{~s}$

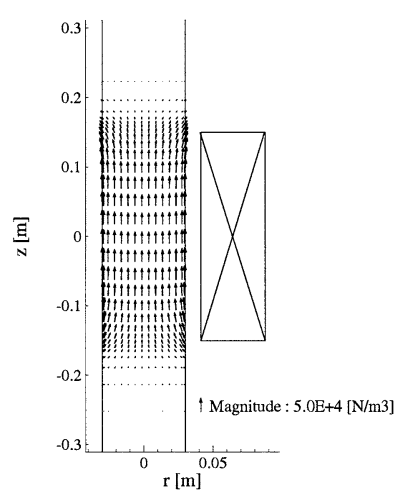

(a) Lorentz force; $F_{r}, F_{z}$

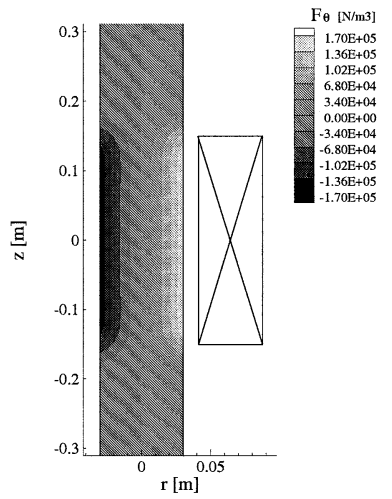

(b) Lorentz force; $F_{\theta}$
Fig. 5 Distribution of Lorentz force at $t=0.01 \mathrm{~s}$

pump and the proposed machine works as a pump.

Figure 5 shows the distribution of Lorentz force near the stator. The $z$-component of Lorentz force $F_{z}$ at $z=0$ is almost constant in the cross section of the duct. On the other hand, $F_{\theta}$ increases with $r$ linearly. For example, $F_{\theta}$ is the same value as $F_{z}$ at $r=10 \mathrm{~mm}$, while it is three times bigger than $F_{z}$ at the duct wall. In the present simulation, the wave number $k$ is the same as that in the experiment, that is $k=20.6 \mathrm{~m}^{-1}\left(39^{\circ}\right.$ at $r=40 \mathrm{~mm}, 52^{\circ}$ at $r=62.5 \mathrm{~mm})^{(8)}$. In order to obtain the maximum axial driving force, therefore, it is necessary to use the coil with bigger twist angle and to replace some amount of $F_{\theta}$ to $F_{z}$.

Figure 6 shows velocity of molten gallium near the stator. The similar phenomenon to Lorentz force appears. Swirl velocity $u_{\theta}$ is ten times or more bigger than $u_{z}$ at the duct wall even at the starting condition.

Figure 7 (a) indicates the induced current in the Hartmann layer at $r=29.9 \mathrm{~mm}$. This figure shows the expansion plan of the cylinder with $r=29.9 \mathrm{~mm}$, and the

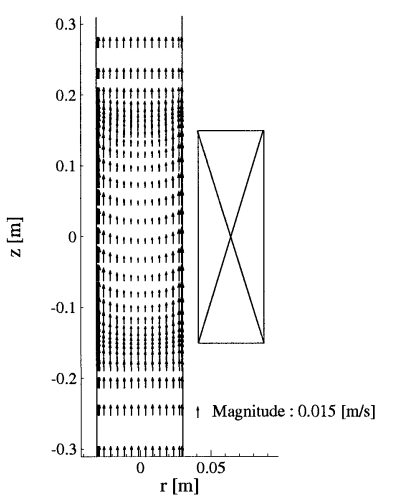

(a) Velocity; $u_{r}, u_{z}$

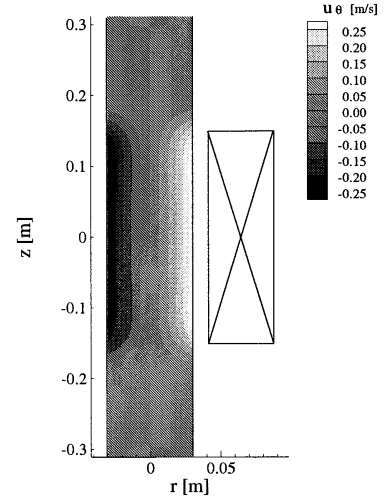

(b) Velocity; $u_{\theta}$
Fig. 6 Distribution of velocity at $t=0.01 \mathrm{~s}$

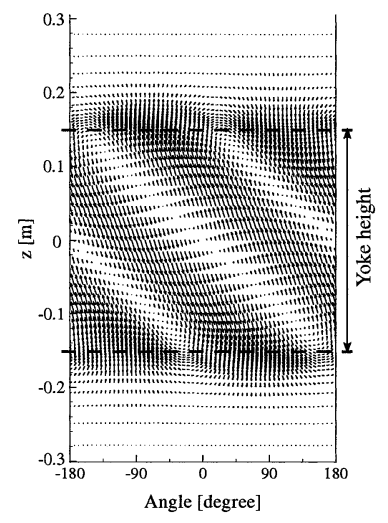

(a) $\theta-z$ distribution in Hartmann boundary layer

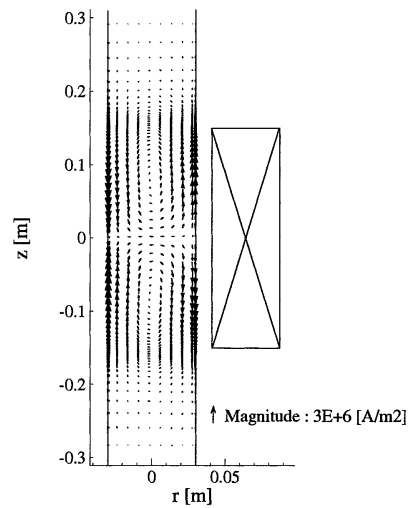

(b) $r-z$ distribution
Fig. 7 Distribution of induced current at $t=0.01 \mathrm{~s}$

horizontal axis is angle and the vertical axis is $z$. Here, the vector is a set of $\left(j_{\theta}, j_{z}\right)$. From Fig. 7 (a), it is confirmed that the induced current in the molten gallium is spiral. Figure 7 (b) shows the induced current in the vertical cross section of the duct. The maximum value of $j_{z}$ is at the duct wall. And also, $j_{\theta}$ has the maximum value at the duct wall although it is not shown in the figure. Comparing these values between $j_{z}$ and $j_{\theta}$ at the duct wall, we can see that $j_{z}$ producing $F_{\theta}$ is about three times bigger than $j_{\theta}$ producing $F_{z}$.

4.2.2 Results at quasi-steady state Table 2 shows the results at quasi-steady state obtained by the present simulation. The flow rate of the numerical result agrees with the experimental result within an error of $18 \%$. This overestimation is caused by the fact that the numerical simulation does not accurately take account of the energy dissipation in turbulence. Besides, the result of the maximum circumferential component of velocity $U_{\theta}$ agrees with the predicted value obtained from the measurement within an error of $40 \%$. This result suggests the validity the slip-pressure function given in the previous pa$\operatorname{per}^{(8)}$. 
Table 2 Result of numerical simulation at $50 \mathrm{~Hz}$

\begin{tabular}{|c|c|c|c|c|}
\hline & $\begin{array}{c}\text { Current } \\
\bar{I} \\
{[\mathrm{~A}]} \\
\end{array}$ & $\begin{array}{c}\text { Flow rate } \\
Q \\
{[\ell / \mathrm{min}]} \\
\end{array}$ & $\begin{array}{c}\text { Pressure } \\
\Delta p \\
{[\mathrm{kPa}]} \\
\end{array}$ & $\begin{array}{c}\text { Maximum Velocity } \\
U_{\theta} \\
{[\mathrm{m} / \mathrm{s}]} \\
\end{array}$ \\
\hline Measurement ${ }^{\dagger}$ & 457 & $8.46 \pm 0.3 \%$ & $5.91 \pm 8 \%$ & $(3.55)$ \\
\hline Numerical simulation & $457^{\S}$ & 9.96 & 8.84 & 5.13 \\
\hline
\end{tabular}

$\dagger$ Reference (8).

$\S$ Actual value in numerical simulation : $457 \mathrm{~A} \times 108 \%$ (see Table 1).

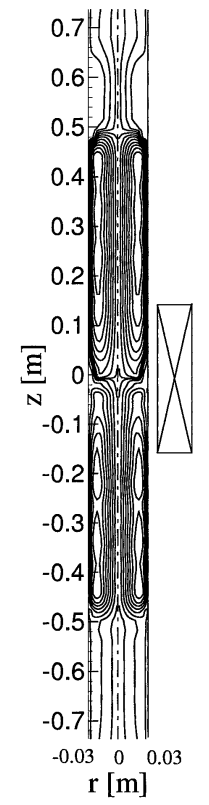

Fig. 8 Streamtubes at quasi-steady state

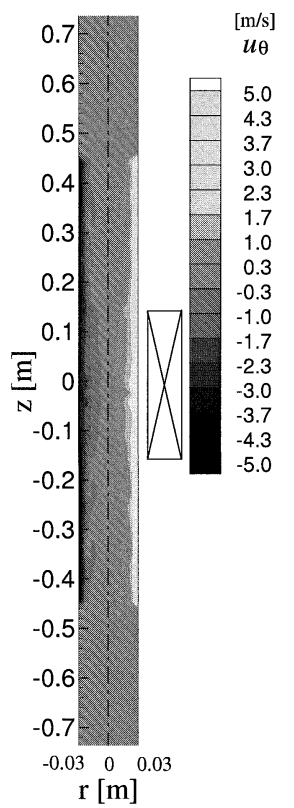

Fig. $9 u_{\theta}$ distribution at quasi-steady state

The streamtubes and the distribution of rotational speed $u_{\theta}$ of molten gallium at quasi-steady condition are shown in Figs. 8 and 9, respectively. Figure 9 shows the swirl flow develops up to the perforated plates at

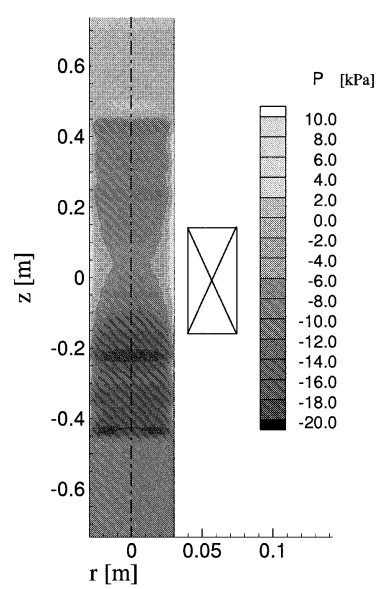

Fig. 10 Pressure distribution at quasi-steady state

$z= \pm 0.46 \mathrm{~m}$. From Fig. 8 , it is confirmed that the excessive spiral flow produces a secondary inward flow in the boundary layer ${ }^{(11)}$ along each perforated plate. This secondary flow forms a pair of upper and lower big torus recirculating flows between two perforated plates. Because the torus circulation dominates the molten gallium flow in the longitudinal cross section, the streamline going through the pump is passing complicated course. In addition, the Kelvin-Helmholtz's unstable phenomenon ${ }^{(12)}$ is seen in Fig. 9.

The pressure distribution in the duct is shown in Fig. 10. Since the excessive swirl flow develops, its centrifugal force produces high pressure at the duct wall and low pressure around the central axis. This pressure distribution is similar to that of a centrifugal pump, however the proposed system functions as an axial pump producing an axial flowrate.

4.2.3 Electromagnetic energy conversion at quasisteady state The electromagnetic energy which is consumed in molten metal per unit time and unit volume is written as follows:

$$
w^{(e m)}=\boldsymbol{j} \cdot \boldsymbol{E} .
$$

Using the extended Ohm's equation, $\boldsymbol{j}=\sigma(\boldsymbol{E}+\boldsymbol{u} \times \boldsymbol{B})$, Eq. (25) is rewritten as ${ }^{(13)}$

$$
\boldsymbol{j} \cdot \boldsymbol{E}=\frac{\boldsymbol{j} \cdot \boldsymbol{j}}{\sigma}+\boldsymbol{u} \cdot(\boldsymbol{j} \times \boldsymbol{B}),
$$

where the first term and the second term of right-hand side of Eq. (26) indicate the Joule heating consumed in molten gallium and the mechanical energy which is supplied to 
the molten gallium, respectively. Figures 11 and 12 show distributions of Joule heating and the mechanical energy production per unit time and unit volume in molten gallium, respectively. Along the central axis of the upper side, there is a region where the energy conversion is negative value as shown in Fig. 12. It is owing to backward flow in the torus recirculating flow. This phenomenon is one of disadvantages of the excessive swirl flow.

The supplied electric power $P$ is converted in the primary coil as follows.

$$
P=P_{J 1}+P_{\text {iron }}+W^{(e m)},
$$

where $P_{J 1}, P_{\text {iron }}$ and $W^{(\mathrm{em})}$ are the Joule heating in the primary coil windings, the iron loss in the yoke, and energy transfer to the secondary conductor per unit time, respectively. In the molten gallium of the whole computation area, the electric energy $W^{(\mathrm{em})}$ is converted as follows:

$$
W^{(e m)}=P_{J 2}+P_{M},
$$

where the electric energy conversion $W^{(e m)}$, the Joule heating $P_{J 2}$ and the mechanical energy production $P_{M}$ per unit time are given by

$$
\begin{aligned}
& W^{(e m)}=\iiint \boldsymbol{j} \cdot \boldsymbol{E} d V, \\
& P_{J 2}=\iiint \frac{\boldsymbol{j} \cdot \boldsymbol{j}}{\sigma} d V, \\
& P_{M}=\iiint \boldsymbol{u} \cdot(\boldsymbol{j} \times \boldsymbol{B}) d V,
\end{aligned}
$$

respectively. Moreover, the mechanical energy production per unit time $P_{M}$ is converted as follows:

$$
P_{M}=Q \Delta p+P_{v}+P_{p},
$$

where $Q \Delta p, P_{v}$ and $P_{p}$ are the mechanical power output of the pump, the viscous dissipation energy in molten gallium flow and that in the perforated plates, respectively.

Table 3 shows each energy conversions in the numerical simulation and those in the experiment ${ }^{(8)}$. The value of the iron loss is not clear in the experiment, but $W^{(\mathrm{em})}$ of numerical simulation suggests the iron loss is in the same order as $W^{(\mathrm{em})}$. The simulation result shows that the out-

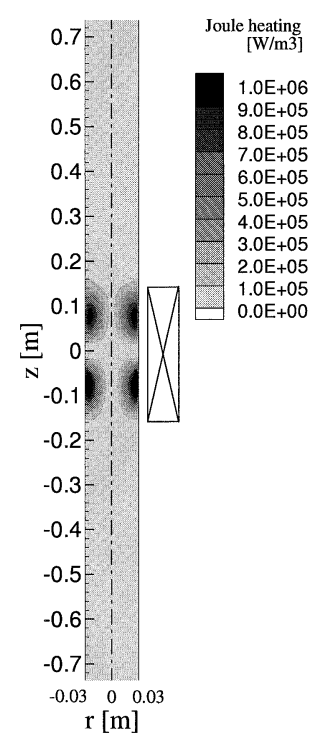

Fig. 11 Joule heating in molten gallium at quasi-steady state

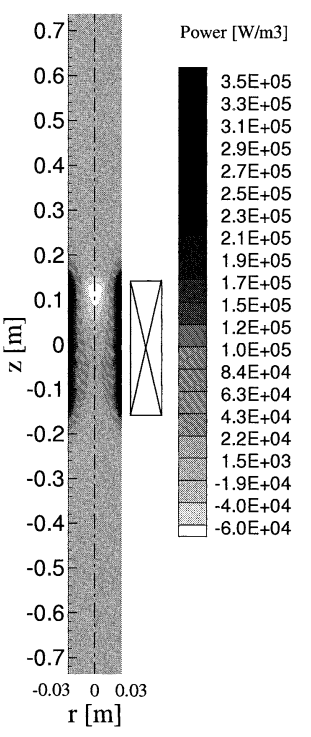

Fig. 12 Mechanical energy production in molten gallium at quasi-steady state

Table 3 Energy conversion in MHD induction pump using rotating twisted magnetic field (at $50 \mathrm{~Hz}$ )

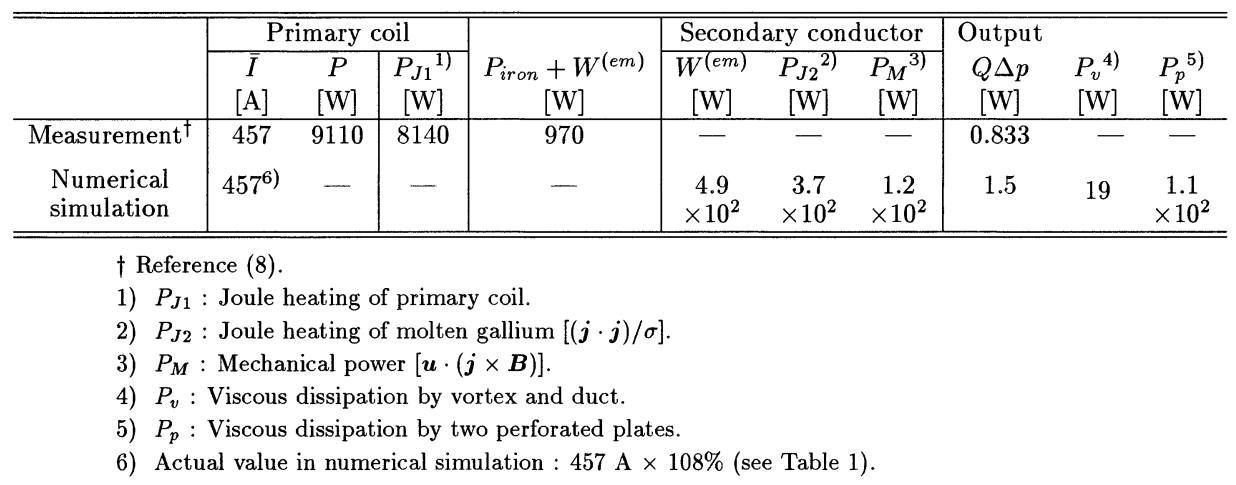


put power $Q \Delta p$ uses only about $1 \%$ of the mechanical energy production $P_{M}$. On the other hand, the perforated plates, which are mounted in the duct system to eliminate the swirl effect, waste most of the mechanical energy.

\section{Conclusions}

The numerical simulation at the same condition as the experiment $\bar{I}=50 \mathrm{~Hz}$ and $f=457 \mathrm{~A}$ was carried out under the magnetic Stokes approximation on $R_{\omega} \ll 1$. Using the computational magnetohydrodynamics, we can obtain the following knowledge that we could not grasp in the experiment.

1. It is confirmed that the proposed induction MHD pump causes the spiral induced current in a molten metal and produces an axial and rotating flow.

2. In the case that the twist angle of the coil is the same as that in the experimental condition, $F_{\theta}$ is three times bigger than $F_{z}$ at a starting condition of $t=0.01 \mathrm{~s}$. In order to obtain the maximum axial driving force, therefore, it is necessary to use the coil with bigger twist angle and to replace some amount of $F_{\theta}$ to $F_{z}$.

3. At quasi-steady state, it is confirmed that the centrifugal force by the excessive spiral flow produces high pressure at the duct wall and low pressure around the central axis.

4. At quasi-steady state, it is confirmed that the excessive spiral flow produces a pair of upper and lower big torus circulation flow between two perforated plates. The torus circulation dominates the molten metal flow in the longitudinal cross section and the streamline going through the pump is passing complicated course.

5. The simulation result at quasi-steady state shows that the mechanical power output $Q \Delta p$ of the pump uses only about $1 \%$ of the mechanical energy production in the molten gallium. It suggests that the perforated plates, which are mounted in the duct system to eliminate the swirl effect, waste most of the mechanical energy.

In order to obtain the maximum power output and the maximum energy efficiencies, it is required that the excessive spiral flow is suppressed without using the perforated plates. In addition, it is also necessary to optimize the twist angle of the coil and the length of the stator.

\section{Acknowledgement}

This research was partially supported by the Ministry of Education, Culture, Sports, Science and Technology, Grant-in-Aid for Young Scientists (A), No.14702053.

\section{Appendix}

\section{A. Boundary condition of the applied magnetic field by the current sheet with coil-end}

Now, we perform the numerical analysis of the applied magnetic field that is generated by the current sheet with coil-end so that the computational analysis simulation appropriately simulates the experimental condition. Hence, we adapt the current sheet at the surface on the yoke, as shown in Fig. 3. The following sections derive the boundary condition of the applied magnetic field which is generated by the current sheet with coil-end.

A.1 Current sheet on the inner surface of the yoke

The sheet current density $\boldsymbol{i}$ (unit; $[\mathrm{A} / \mathrm{m}]$ ) in the working coil part $\left(r=a_{1},-z_{1} \leq z \leq z_{1}\right)$ is expressed as follows ${ }^{(6)}$ :

$$
\left\{\begin{array}{l}
i_{r}=0 \\
i_{\theta}=\sqrt{2} \bar{I} N k \cos (\omega t-\theta-k z), \\
i_{z}=-\frac{\sqrt{2} \bar{I} N}{a_{1}} \cos (\omega t-\theta-k z),
\end{array}\right.
$$

where $\bar{I}$ is the effective current supplied from the power source, $N$ is the windings number, $k$ is the wave number of $z$-direction $(k=2 \pi / \lambda)$ and $\omega$ is angular frequency [rad/s].

\section{A.2 Current sheet on the upper and the lower sur- faces of the yoke}

The preconditions of the current sheet in the upper surface of the yoke are expressed as

$$
\begin{cases}r=a_{0} \quad: \quad i_{r}=0 \\ r=a_{1} \quad: \quad i_{r}=-\frac{\sqrt{2} \bar{I} N}{a_{1}} \cos \left(\omega t-\theta-k z_{1}\right), \\ a_{1} \leq r \leq a_{0}: & \left|i_{r}\right| \text { is a monotonous decrease, } \\ & i_{z}=0 .\end{cases}
$$

The following current sheets satisfy the precondition (34) and $\nabla \cdot \boldsymbol{i}=0$ :

$$
\left\{\begin{array}{l}
i_{r}=-\frac{\sqrt{2} \bar{I} N}{a_{1}} \frac{\sqrt{a_{0}}-\sqrt{r}}{\sqrt{a_{0}}-\sqrt{a_{1}}} \cos \left(\omega t-\theta-k z_{1}\right), \\
i_{\theta}=\frac{\sqrt{2} \bar{I} N}{a_{1}} \frac{\frac{3}{2} \sqrt{r}-\sqrt{a_{0}}}{\sqrt{a_{0}}-\sqrt{a_{1}}} \sin \left(\omega t-\theta-k z_{1}\right), \\
i_{z}=0 .
\end{array}\right.
$$

The $\theta$-component of the current sheet $i_{\theta}$ is a monotonous increase in the range of $a_{1} \leq r \leq a_{0}\left(a_{0}=90 \mathrm{~mm}, a_{1}=\right.$ $62.5 \mathrm{~mm}$ ).

Similarly, we can obtain the current sheets in the lower surface of the yoke as follows:

$$
\left\{\begin{array}{l}
i_{r}=\frac{\sqrt{2} \bar{I} N}{a_{1}} \frac{\sqrt{a_{0}}-\sqrt{r}}{\sqrt{a_{0}}-\sqrt{a_{1}}} \cos \left(\omega t-\theta+k z_{1}\right), \\
i_{\theta}=-\frac{\sqrt{2} \bar{I} N}{a_{1}} \frac{\frac{3}{2} \sqrt{r}-\sqrt{a_{0}}}{\sqrt{a_{0}}-\sqrt{a_{1}}} \sin \left(\omega t-\theta+k z_{1}\right), \\
i_{z}=0 .
\end{array}\right.
$$

\section{A.3 Boundary condition of the applied magnetic field in the surface of the stator}

The integral representation of Ampére's law is applied to a closed loop across the current sheet. Assuming an ideal yoke $[\mu]_{\text {yoke }} / \mu_{0} \gg 1$, we obtain the tangential component of the magnetic flux density on the surface of the yoke ${ }^{(6)}$. The obtained boundary conditions are written in the followings. 
- Inner surface of the yoke: $-z_{1} \leq z \leq z_{1}, r=a_{1}$,

$$
\begin{aligned}
& {\left[B_{\theta}\right]_{\text {gap }}=\frac{\sqrt{2} \mu_{0} \bar{I} N}{a_{1}} \cos (\omega t-\theta-k z),} \\
& {\left[B_{z}\right]_{\text {gap }}=\sqrt{2} \mu_{0} \bar{I} N k \cos (\omega t-\theta-k z) .}
\end{aligned}
$$

- Upper surface of the yoke: $z=z_{1}, a_{1} \leq r \leq a_{0}$,

$$
\begin{aligned}
& {\left[B_{r}\right]_{\text {gap }}=\frac{\sqrt{2} \mu_{0} \bar{I} N}{a_{1}} \frac{\frac{3}{2} \sqrt{r}-\sqrt{a_{0}}}{\sqrt{a_{0}}-\sqrt{a_{1}}} \sin \left(\omega t-\theta-k z_{1}\right),} \\
& {\left[B_{\theta}\right]_{\text {gap }}=\frac{\sqrt{2} \mu_{0} \bar{I} N}{a_{1}} \frac{\sqrt{a_{0}}-\sqrt{r}}{\sqrt{a_{0}}-\sqrt{a_{1}}} \cos \left(\omega t-\theta-k z_{1}\right) .}
\end{aligned}
$$

- Lower surface of the yoke: $z=-z_{1}, a_{1} \leq r \leq a_{0}$,

$$
\begin{aligned}
& {\left[B_{r}\right]_{\text {gap }}=\frac{\sqrt{2} \mu_{0} \bar{I} N}{a_{1}} \frac{\frac{3}{2} \sqrt{r}-\sqrt{a_{0}}}{\sqrt{a_{0}}-\sqrt{a_{1}}} \sin \left(\omega t-\theta+k z_{1}\right),} \\
& {\left[B_{\theta}\right]_{\text {gap }}=\frac{\sqrt{2} \mu_{0} \bar{I} N}{a_{1}} \frac{\sqrt{a_{0}}-\sqrt{r}}{\sqrt{a_{0}}-\sqrt{a_{1}}} \cos \left(\omega t-\theta+k z_{1}\right) .}
\end{aligned}
$$

\section{References}

(1) Garnier, M., Technological and Economical Challenges Facing EPM in the Next Century, Proc. 3rd Int. Symp. on Electromagnetic Processing of Materials, (2000), pp.3-8.

( 2 ) Fujii, T., State of Art Electromagnetic Processing in Japanese Iron and Steel Industry, Proc. 3rd Int. Symp. on Electromagnetic Processing of Materials, (2000), pp.14-19.

( 3 ) Blake, L.R., Conduction and Induction Pumps for Liquid Metals, Proc. Inst. Elec. Eng., (1956), pp.49-63.

(4) Arazeki, H., Kirillov, I.R., Preslitsky, G.V. and Ogorodnikov, A.P., Double-Supply-Frequency Pres- sure Pulsation in Annular Linear Induction Pump, Part II: Reduction of Pulsation by Linear Winding at Both Stator Ends, Nuclear Engineering and Design, Vol.195 (2000), pp.397-406.

( 5 ) Ueno, K., Ando, T., Taniguchi, S. and Takagi, T., Axial Driving Force Induced by Rotating Twisted Magnetic Field, Proc. 3rd Int. Symp. on Electromagnetic Processing of Materials, (2000), pp.491-496.

(6) Ando, T., Ueno, K., Taniguchi, S. and Takagi, T., Induction Pump for High-Temperature Molten Metals Using Rotating Twisted Magnetic Field: Thrust Measurement Experiment with Solid Conductors, IEEE Trans. Magnetics, Vol.38, No.4 (2002), pp.1789-1796.

( 7 ) Ando, T., Ueno, K., Taniguchi, S. and Takagi, T., Visual System Experiment of MHD Pump Using Rotating Twisted Magnetic Field Applicable to HighTemperature Molten Metals, ISIJ Int., Vol.43 (2003), pp.849-854.

( 8 ) Ando, T., Ueno, K., Taniguchi, S. and Takagi, T., Induction Pump for High-Temperature Molten Metals Using Rotating Twisted Magnetic Field: Molten Gallium Experiment, IEEE Trans. Magnetics, Vol.40, No.4 (2004), pp.1846-1857.

(9) Harlow, F.H. and Welch, J.E., Numerical Calculation of Time-Dependent Viscous Incompressible Flow of Fluid with Free Surface, The Physics of Fluids, Vol.8 (1965), pp.2182-2189.

(10) Kawamura, T. and Kuwahara, K., Computation of High Reynolds Number Flow around a Circular Cylinder with Surface Roughness, AIAA paper 84-0340, (1984).

(11) Schlichting, H., Boundary-Layer Theory 7th Ed., (1978), pp.225-230, McGraw-Hill.

(12) Japan Society of Fluid Mechanics, Ed., Handbook of Fluid Mechanics 2nd Ed., (in Japanese), (1998), p.166, Maruzen.

(13) Imai, I., Thinking Electromagnetics, (in Japanese), (1990), pp.69-70, Saiensu-Sya. 